\title{
The Effect of Metallic Ions on the Enhanced Upconversion Emission of $\mathrm{NaGdF}_{4}$ Nanostructures $^{2}$
}

\author{
Deepthi Muraleedharan, Jin Zhang* \\ ${ }^{1}$ Department of Chemical and Biochemical Engineering, University of Western Ontario \\ London, Ontario, N6A 5B9, Canada \\ dmuralee@uwo.ca; jzhang@eng.uwo.ca
}

\begin{abstract}
Co-doping of $\mathrm{NaGdF}_{4}$ doped with $\mathrm{Yb}^{3+}$ and $\mathrm{Er}^{3+}$ with $\mathrm{Fe}^{3+}$ have shown to improve the intensity of upconversion emissions of green and red wavelengths. The maximum emission intensity was obtained at a Fe concentration of $0.3 \mathrm{mmols}$ in the precursor and using a laser excitation at $980 \mathrm{~nm}$ wavelength. The green emission was found to be enhanced to 10 times and red emissions was enhanced to about 3 times as compared to the intensity of the emissions without the Fe co-doping. Characterisation techniques like XRD and EDS is used to investigate the reason for this observation.
\end{abstract}

Keywords: Upconversion nanostructures, photoluminescence, metallic ion, doping

\section{Introduction}

There are several research findings on the improvement of luminance emission intensity on co-doping with a third ion. Co-doping of $\mathrm{NaGdF}_{4}$ doped with $\mathrm{Yb}^{3+}$ and $\mathrm{Er}^{3+}$ with $7 \mathrm{~mol} \%$ of $\mathrm{Li}^{+}$ions increases the luminescence intensity of green emissions by 47 fold and red emissions by 23 fold [1] and co-doping with $30 \mathrm{~mol} \% \mathrm{Ca}^{2+}$ improves the emission intensity by 200 times [2]; codoping with $13.5 \mathrm{~mol} \%$ of $\mathrm{Mg}^{2+}$ increases luminescence intensity of green and red emissions by 6 and 9 times respectively [3] (Shuwen Zhao, 2016). Similarly, in case of $\mathrm{NaYF}_{4}$ doped with $\mathrm{Yb}^{3+}$ and $\mathrm{Er}^{3+}$, co-doping with $0.5 \mathrm{~mol}$ $\%$ of $\mathrm{Li}^{+}$produced green emission of 34 fold intensity and red emission of 101 fold intensity [4]; But co-doping with 10mol $\% \mathrm{Mo}^{3+}$, the green emission and red emissions became 6 fold and 8 fold respectively [5]; with $15 \mathrm{~mol} \% \mathrm{Cr}^{3+}$ green emissions and red emission became 16 and 7 times [6] and with $20 \mathrm{~mol} \% \mathrm{La}^{3+}$, green and red emission intensity became 7.6 and 8.1 times respectively (Junxiang Fu, 2017) and with $7 \mathrm{~mol} \%$ of $\mathrm{Cd}^{2+}$ total emission intensity increased 38 times [7]. Yahong Hu et al. shows that the red emission luminance intensity of $\mathrm{NaYF}_{4}: \mathrm{Yb}^{3+}, \mathrm{Er}^{3+}$ becomes 14 -fold on adding $10 \mathrm{~mol} \%$ of $\mathrm{Mo}^{3+}$ and 24 fold on adding 30mol \% Fe ${ }^{3+}$ [8]. 20mol \% co-doping with $\mathrm{Fe}^{3+}$ showed 7 times increase in red emission [9]. Tie Cong et al. shows that the emission intensities of green and red increased at first and then decreased with the increase of concentration of $\mathrm{Zn}^{2+}$ from 0 to $20 \mathrm{~mol} \%$ [10].

\section{Materials and Experimental}

\subsection{Materials}

Gadolinium(III) nitrate hexahydrate $\left(\mathrm{Gd}\left(\mathrm{NO}_{3}\right)_{3} \cdot 6 \mathrm{H}_{2} \mathrm{O}\right)$, ytterbium(III) nitrate pentahydrate $\left(\mathrm{Yb}\left(\mathrm{NO}_{3}\right)_{3} \cdot 5 \mathrm{H}_{2} \mathrm{O}\right)$, erbium(III) nitrate pentahydrate $\left(\mathrm{Er}\left(\mathrm{NO}_{3}\right)_{3} \cdot 5 \mathrm{H}_{2} \mathrm{O}\right)$, Iron(III) nitrate nonahydrate $\left(\mathrm{Fe}\left(\mathrm{NO}_{3}\right)_{3} \cdot 9 \mathrm{H}_{2} \mathrm{O}\right)$ sodium Fluoride $(\mathrm{NaF})$, branched polyethylenimine (PEI) and ethylene glycol (EG) purchased from Sigma-Aldrich and deionized water was used for the synthesis of nanostructures.

\subsection{Synthesis of Metallic lons Doped Nagdf 4 Nanoparticles}

Modified solvothermal method [11] was used in the synthesis of $\mathrm{NaGdF}_{4}$ doped with $\mathrm{Yb}$, Er and varied amounts of $\mathrm{Fe}$. $1.6 \mathrm{mmol}$ Gadolinium(III) nitrate hexahydrate, $0.38 \mathrm{mmol}$ Erbium(III) nitrate pentahydrate, $0.36 \mathrm{mmol}$ Ytterbium(III) nitrate pentahydrate, 0 to $0.8 \mathrm{mmol}$ of Iron(III) nitrate nonahydrate and $0.7 \mathrm{~g}$ polyethylenimine are added to into $20 \mathrm{~mL}$ ethylene Glycol in a three-neck round bottom flask and dispersed by stirring at room temperature. 8 mmol Sodium fluoride is added to $10 \mathrm{ml}$ Ethylene Glycol and sonicated to ensure a clear solution. This solution is added to the three-neck round bottom flask dropwise while continuing to still with the help of magnetic stirrer and temperature is increased to $198{ }^{\circ} \mathrm{C}$ refluxing for 6 
hours under nitrogen atmosphere. After the completion of the reaction, the mixture is centrifuged and washed with a 50:50 mixture of ethanol and water three times. The resulting nano structures are dried overnight at $60^{\circ} \mathrm{C}$.

\subsection{Characterization of $\mathrm{Nagdf}_{4}$ Nanoparticles Doped With Metallic Ions}

The size and morphology of nanostructures were characterized by transmission electron microscopy (TEM, Philips CM10 operated at $80 \mathrm{kV}$ ). The crystal structures of NaGdF4-based nanostructures were studied by X-ray diffraction (XRD, Rigaku rotating-anode X-ray diffractometer with $\mathrm{Co}-\mathrm{K} \alpha$ radiation). The fluorescent emission spectrum of NaGdF4-based nanostructures were measured by fluorophotometry (QuantaMsterTM 30, Horiba Canada-PTI) with an external excitation wavelength of $980 \mathrm{~nm}, 1 \mathrm{~W}$.

\section{Results and Discussion}

Typical green $\left(\lambda_{\mathrm{em}}=540 \mathrm{~nm}\right)$ and red $\left(\lambda_{\mathrm{em}}=655 \mathrm{~nm}\right)$ emissions of $\mathrm{Er}^{3+}$ can be observed in all the samples using an excitation wavelength, $\lambda_{\text {ex' }}$ of $980 \mathrm{~nm}$. It is found that in most of the samples the green emission is less than $50 \%$ of red emissions.

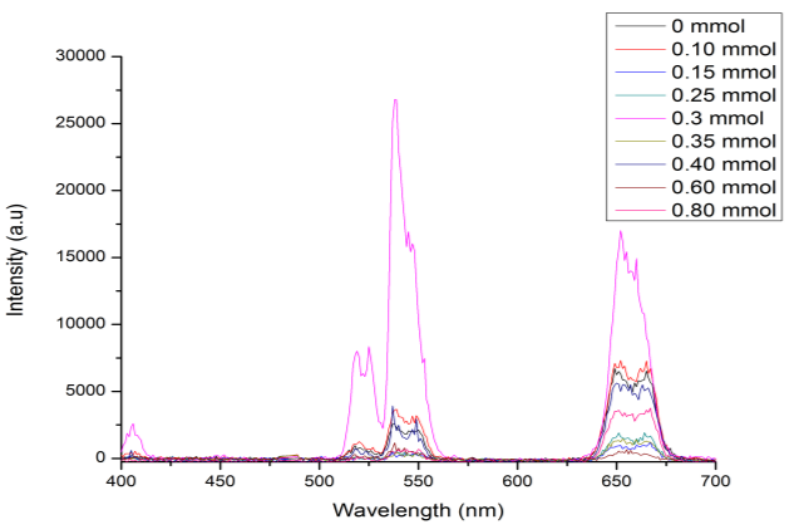

Figure 1. Upconversion luminescence properties of NaGdF4 doped without/ with $\mathrm{Yb}^{3+}$, $\mathrm{Er}^{3+}$ and $\mathrm{Fe}^{3+}(0-0.8 \mathrm{mmol})$ under 980nm excitation wavelength $\left(\lambda_{\mathrm{ex}}\right)$.

As compared to the rest of the emission graphs, sample containing $0.3 \mathrm{mmol} F e$ has higher green to red ratio and overall higher intensity of emissions. The green emission is enchanced to about 10 times and the red emission is enchanced to about 3 times at this iron concentration.

Erbium content is seen to be minimum in $0.3 \mathrm{mmol} F e$ containing sample. Previous studies also suggest that higher Er3+ results in lower upconversion intensity due to quenching effect [12]. This explains the highest overall intensity of emission for sample containing $0.3 \mathrm{mmol}$ Fe.

The intensity of upconversion emission is known to be directly proportional to the $\mathrm{n}$ th power of input power, with $\mathrm{n}$ being the average number of photon absorption resulting in the emission [13].

$I \approx P^{n}$

Where $\mathrm{I}$ is the intensity, $\mathrm{P}$ the laser power and $\mathrm{n}$ the number of photons absorbed for emission of one photon. The value of $\mathrm{n}$ gives the number of photons involved in the emission of particular wavelength peaks. 

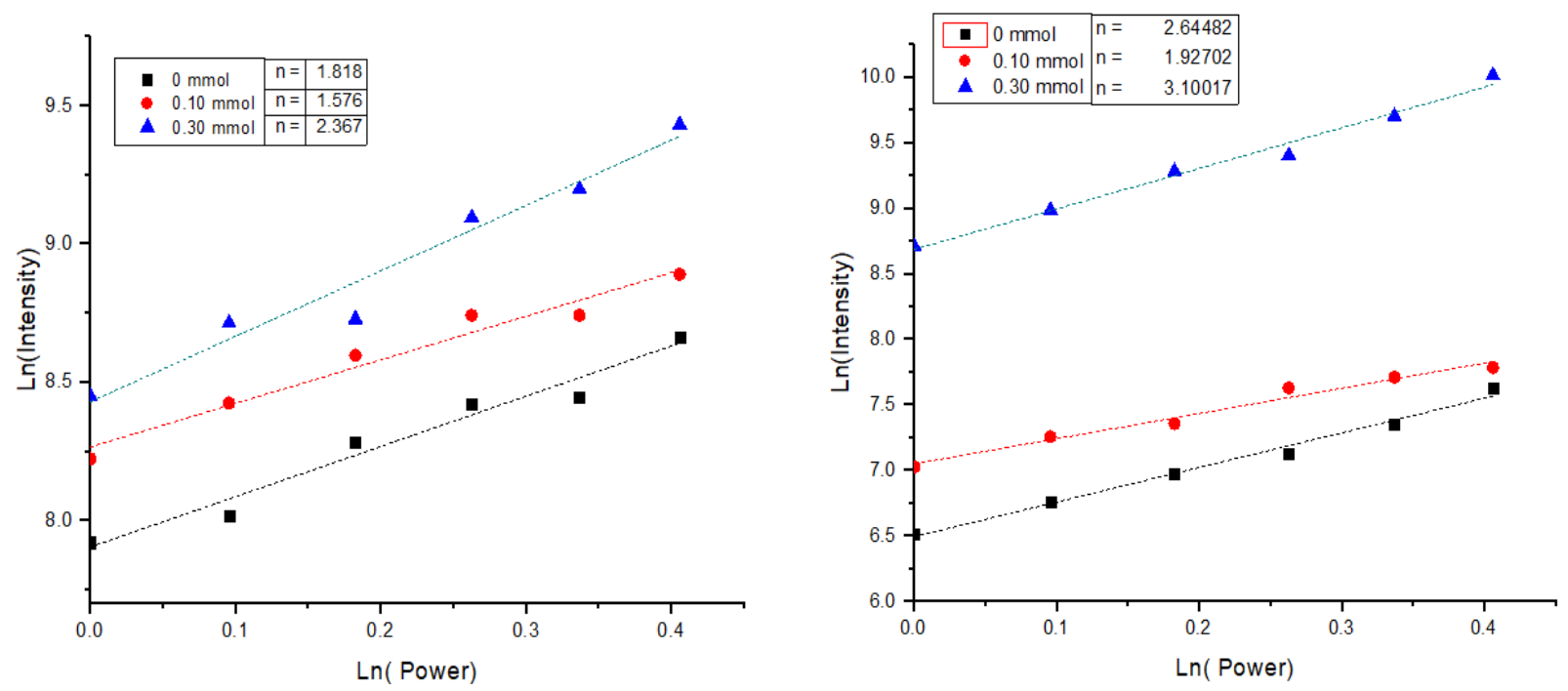

Figure 2. Log intensity vs log power plot for different precursor iron content from $0 \mathrm{mmol}$ to $0.3 \mathrm{mmol}$ of (a) red peak $(650 \mathrm{~nm})$ and (b) green peak $(537 \mathrm{~nm})$

The red emission is found to be the result of the following transitions utilizing two photons $\mathrm{Yb} 3+(2 \mathrm{~F} 5 / 2 \rightarrow 2 \mathrm{~F} 7 / 2)$ and $\mathrm{Er} 3+(4 \mathrm{I} 15 / 2 \rightarrow 4 \mathrm{I} 11 / 2), \mathrm{Er} 3+(4 \mathrm{I} 11 / 2 \rightarrow 4 \mathrm{I} 13 / 2)$ and $\mathrm{Yb} 3+(2 \mathrm{~F} 5 / 2 \rightarrow 2 \mathrm{~F} 7 / 2)$ and $\mathrm{Er} 3+(4 \mathrm{I} 13 / 2 \rightarrow 4 \mathrm{I} / 2)$. The green emisssions are result of $\mathrm{Yb} 3+(2 \mathrm{~F} 5 / 2 \rightarrow 2 \mathrm{~F} 7 / 2)$ and $\mathrm{Er} 3+(4 \mathrm{I} 15 / 2 \rightarrow 4 \mathrm{I} 11 / 2)$ and $\mathrm{Yb} 3+(2 \mathrm{~F} 5 / 2 \rightarrow 2 \mathrm{~F} 7 / 2)$ and $\mathrm{Er} 3+(4 \mathrm{I} 11 / 2$ $\rightarrow 2 \mathrm{H} 11 / 2)$. This is two photon emission. But at higher power $\mathrm{Yb} 3+(2 \mathrm{~F} 5 / 2 \rightarrow 2 \mathrm{~F} 7 / 2)$ and $\mathrm{Er} 3+(4 \mathrm{~F} 9 / 2 \rightarrow 2 \mathrm{H} 9 / 2)$ or $\mathrm{Yb} 3+$ $(2 \mathrm{~F} 5 / 2 \rightarrow 2 \mathrm{~F} 7 / 2)$ and $\mathrm{Er} 3+(4 \mathrm{I} 9 / 2 \rightarrow 2 \mathrm{H} 9 / 2)$ followed by non radiative transition Er3+ $(2 \mathrm{H} 9 / 2 \rightarrow 2 \mathrm{H} 11 / 2)$ also can result in green emissions (F.Song, 1999). This explains the possibility of value $n=3$ (Figure 22).

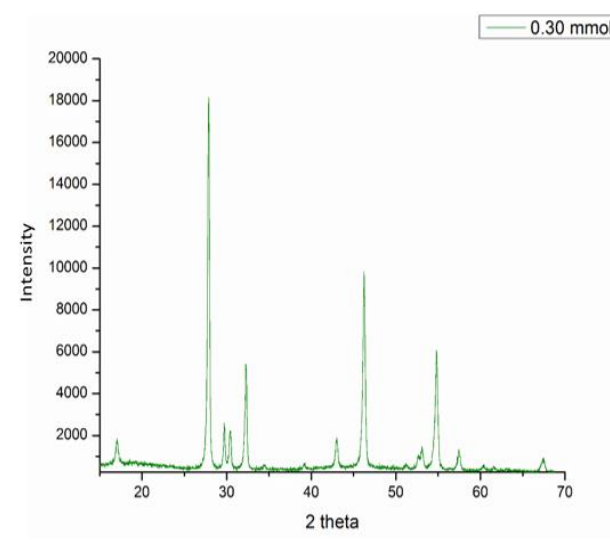

Figure 3. XRD spectra of NaGdF4 doped with $\mathrm{Yb} 3+, \mathrm{Er}^{3+}$ and $\mathrm{Fe}^{3+}(0.3 \mathrm{mmol})$

Samples expect $0.3 \mathrm{mmol} \mathrm{Fe}$ is found to be cubic lattice structure. $0.3 \mathrm{mmol} \mathrm{Fe}$ is found to be a mixture of cubic and hexagonal lattice structure. NaGdF4 doped with $\mathrm{Yb} 3+, \mathrm{Er} 3+$ and $\mathrm{Fe} 3+$ with hexogonal shaped lattice is found to have higher green emission compared to red emission [14] (Parthiban Ramasamy, 2013). Doping of Iron can induce phase changes. 


\section{CONCLUSION}

Upconversion nanostructures with optical and magnetic properties were prepared using solvothermal process. The lower external magnetic field applied did not show considerable influence of optical properties of the UCNP. Co-doping with Iron is found to influence the optical emission of NaGdF4 doped with $\mathrm{Yb} 3+$, Er3+. The higher iron content in the nanostructure resulted in higher over all emissions, higher green to red ratio compared to the rest of the samples and have shown transition in crystal structure from pure cubic to a mixture of cubic and hexogonal lattice structures. The maximum emission intensity was obtained at a Fe concentration of $0.3 \mathrm{mmols}$ in the precursor and using a laser excitation at $980 \mathrm{~nm}$ wavelength. The green emission was found to be enhanced to 10 times and red emissions was enhanced to about 3 times as compared to the intensity of the emissions without the Fe co-doping.

\section{Acknowledgements}

Authors are thankful for the financial support from Canada Innovation Fund.

\section{References}

[1] Qian Cheng, J. S. (2012). Enhanced upconversion emission in Yb3+ and Er3+ codoped NaGdF4 nanocrystals by introducing Li+ ions. Nanoscale, 779-784.

[2] Lei Lei, D. C. (2014). Highly Intensified Upconversion Luminescence of Ca2+-doped Yb/Er:NaGdF4 Nanocrystals Prepared by a Solvothermal Route. Asian Journal of Chemistry, 728 - 733.

[3] Shuwen Zhao, W. L. (2016). Enhanced upconversion luminescence and modulated paramagnetic performance in NaGdF4:Yb, Er by Mg2+ tridoping. The Royal Society of Chemistry, 81542-81551.

[4] Xiuwen Wang, $\uparrow . X$. (2013). Comprehensive Studies of Li+ Effect on NaYF4:Yb/Er Nanocrystals:Morphology, Structure, and Upconversion Luminescence. Royal Society of Chemistry, 1-3.

[5] Dongguang Yin, C. W. (2014). Enhancing upconversion luminescence of NaYF4:Yb/Er nanocrystals by Mo3+ doping and their application in bioimaging. The Royal Society of Chemistry, 12037-12043.

[6] Chuanying Wang, X. C. (2015).. Journal of Alloys and Compounds 649, 196-203

[7] Cong, T., Ding, Y., Yu, X., Mu, Y., Hong, X., \& Liu, Y. (2017). Upconversion luminescence enhancement in NaYF4: $\mathrm{Yb} 3+, \mathrm{Er} 3+$ nanoparticles induced by Cd2+ tridoping. Materials Research Bulletin , 151-155.

[8] Yahong Hu, X. L. (2015). Enhancement of the red upconversion luminescence in NaYF4:Yb3+, Er3+ nanoparticles by the transition metal ions doping. Ceramics International, 4545-14553.

[9] Jing Tang, L. C. (2015). Selectively enhanced red upconversion luminescence and phase/size manipulation via Fe3+ doping in NaYF4:Yb,Er nanocrystals. Nanoscale, 14752- 14759.

[10] Tie Cong, Y. D. (2016). Synthesis and optical properties of Zn2+ doped NaYF4: Yb3+, Er3+upconversion nanoparticles. Materials Letters, 59-62.

[11] Longyi Chen, W. H. (2017). Special properties of luminescent magnetic NaGdF4:Yb3+, Er3+ upconversion nanocubes with surface modifications. The Royal Society of Chemistry, 26770-26775.

[12] F.Song, M. S. (1999). Effect of Erbium Concentration on Upconversion Luminescence of Er:Yb:phosphate Glass Exited by InGaAs Laser Diode. S PI E, 1822188.

[13] M. Pollnau, D. R. (n.d.). Power dependence of upconversion luminescence in lanthanide and transition-metal-ion systems.

[14] Parthiban Ramasamy, P. C. ( 2013). Enhanced upconversion luminescence in NaGdF4:Yb,Er nanocrystals by Fe3+ doping and their application in bioimaging. Nanoscale, , 8711-8717. 